\title{
Characterization of Near-Ground Radio Propagation Channel for Wireless Sensor Network with Application in Smart Agriculture ${ }^{+}$
}

\author{
Hicham Klaina ${ }^{1, *}$, Ana Alejos ${ }^{1}$, Otman Aghzout ${ }^{2}$ and Francisco Falcone ${ }^{3}$ \\ 1 Dept. of Signal theory and Communications, University of Vigo, Vigo, Spain; analejos@uvigo.es \\ 2 TITM department, ENSATe, AbdelMalek Essaadi University, Tetouan, Morocco; o.aghzout@gmail.com \\ 3 Dept. of Electronic and Electrical Engineering, Public University of Navarre, Pamplona, Spain; \\ francisco.falcone@unavarra.es \\ * Correspondence: hklaina@uvigo.es; Tel.: +34-695-131-063 \\ + Presented at the 4th International Electronic Conference on Sensors and Applications (ECSA 2017), \\ 15-30 November 2017; Available online: https://sciforum.net/conference/ecsa-4.
}

Published: 14 November 2017

\begin{abstract}
In this contribution, we present a narrowband radio channel model for a scenario wherein the radio link operates under near-ground conditions, occurring on a ZigBee wireless sensor networks applied to smart agriculture. A near-ground network deployment can be useful to avoid tall antenna masts, or once crops grow. Among the examined scenarios, we analyzed path loss caused when placing sensor nodes in soil, short and tall grass fields. We measured the received power when locating both transmitter and receiver antennas at two different heights. The path loss was then estimated as dependent of the radio link range. In another scenario, RSSI were obtained to analyze the communication quality between sensor nodes using same antennas heights as the previous scenarios, only for the case of a short grass field.
\end{abstract}

Keywords: Internet of Things; Wireless Sensor Networks; Smart Agriculture; near-ground; radio channel model.

\section{Introduction}

Wireless Sensor Network technology is based on collecting data from sensor nodes, which communicate between each other and between them and the gateway, which transmit the data through internet for storage, analysis and processing [1,2]. It has attracting applications in variety of fields as health care, machine surveillance and military uses [3]. In the past few years, the WSN has emerged into the research field of agriculture. It provides a feasible way for low-cost, high-efficiency, and high-productivity agriculture farming [4,5]. Some agriculture related parameters, such as soil temperature, soil moisture, $\mathrm{CO} 2, \mathrm{PH}$ value and soil nutrients, could be monitored and then controlled by wireless sensor networks [6]. However, when placing nodes near to the ground in agriculture fields, the transmitted signal experiences a high-level of attenuation due to the components of the field like grass and soil. Path loss caused by near-ground is explained in terms of Fresnel zones $[7,8]$. These losses are reason of bad communication between nodes, thus unsuccessful application since the success of the wireless sensor network applications highly depends on the reliable communication among the sensor nodes. This paper presents a developed channel model, which considers the path loss caused by agriculture fields found in the propagation link when a near ground radio communication occurs. Radio communication, using transmitter and receiver antennas working at $868 \mathrm{MHz}, 2.4 \mathrm{GHz}$ and $5.8 \mathrm{GHz}$, has been implemented in soil, short and tall grass fields. We measured the received power at the receiver antenna at two different heights, $20 \mathrm{~cm}$ and $40 \mathrm{~cm}$ above ground. The path loss was then estimated as dependent of the radio link range. In another 
scenario, we analyzed the communication quality between sensor nodes dedicated to smart agriculture, when placing them at the same two different antenna heights, but only for the case of a short grass field, by measuring the RSSI values. These nodes are working at $2.4 \mathrm{GHz}$ under ZigBee protocol. A comparison between path loss and RSSI results for different antennas heights has been completed. The results have been employed in a context aware environment based on WSN deployment and integration, applied in a Smart Agriculture use case.

\section{Materials and Methods}

\subsection{Indoor Experiment on Near Ground Path Loss in Near Field}

The objective of this experiment is to investigate if the ground will cause path losses on a RF signal propagated in the line of sight between the transmitter and receiver antennas in near field $(<1.5 \mathrm{~m})$ when changing height, by lowering both antennas from $\mathrm{H}_{1}=66.85 \mathrm{~cm}$ to $\mathrm{H}_{2}=52.15 \mathrm{~cm}$, and then to $\mathrm{H}_{3}=31.95 \mathrm{~cm}$. Furthermore, we will see the difference in the path losses when using three different frequency ranges $\mathrm{F}_{1}=868 \mathrm{MHz}, \mathrm{F}_{2}=2.4 \mathrm{GHz}$ and $\mathrm{F}_{3}=5.8 \mathrm{GHz}$. In this experiment, $\mathrm{a}$ transmitted power of $\mathrm{P}_{\mathrm{t}}=6 \mathrm{dBm}$ has been used. Path losses were obtained from the measured received powers using Friis equation (1), and compared to the proposed model using $(2,3)$.

$$
P_{r}=P_{t}+G_{t}+G_{r}-P_{L}
$$

where $P_{t}$ and $P_{r}$ are respectively the transmitted and the received power in $\mathrm{dBm}, G_{t}$ and $G_{r}$ are respectively the transmitter and the receiver antennas gains in $\mathrm{dBi}$.

$$
\begin{gathered}
P_{L}=L_{f} \text { if } d<d_{b}, \\
P_{L}=L_{f}+L_{N G} \text { if } d \geq d_{b},
\end{gathered}
$$

where $L_{f}$ is the free space path loss, $L_{N G}$ is the near-ground path loss in $d B m$ and $d_{b}$ is the minimum distance for the existence of near-ground path loss.

$$
\mathrm{L}_{\mathrm{f}}=32.44+20 \log _{10}\left(\mathrm{f} \times 10^{-6}\right)+20 \log _{10}\left(\mathrm{~d} \times 10^{-3}\right),
$$

Here $\mathrm{f}$ is the transmitted signal frequency in $\mathrm{Hz}$ and $\mathrm{d}$ is the distance between the transmitter and the receiver in $\mathrm{m}$.

$$
\begin{gathered}
\mathrm{L}_{\mathrm{NG}}=-20 \log _{10}\left(\frac{5 \sqrt{\mathrm{h}_{\mathrm{t}} \mathrm{h}_{\mathrm{r}}}}{3 \sqrt{\mathrm{d} \lambda}}+\frac{35 \mathrm{~h}_{\mathrm{t}} \mathrm{h}_{\mathrm{r}}}{6 \mathrm{~d} \lambda}\right), \\
\mathrm{d}_{\mathrm{b}}=\frac{\mathrm{h}_{\mathrm{t}} \mathrm{h}_{\mathrm{r}}}{0.09 \lambda^{\prime}}
\end{gathered}
$$

where respectively $h_{t}$ and $h_{r}$ are the heights of the transmitter and the receiver and $\lambda$ is the wavelength of the transmitted wave [7].

\subsection{Path loss in Agriculture Fields Experiments}

The purpose of this experiment is to observe the effect of soil, short and tall grass on the RF propagation in agriculture fields, by measuring the path loss exist due to both free space and ground while lowering the height of both transmitter and receiver antennas working at $868 \mathrm{MHz}, 2.5 \mathrm{GHz}$ and $5.8 \mathrm{GHz}$.

The first step was to fix both transmitter and receiver on a height of $\mathrm{H}_{2}=0.4 \mathrm{~m}$ from the ground. Measurements have been started with a separation distance of $d_{1}=1 \mathrm{~m}$. The transmitter was kept stationary and the receiver was moved each time with $2 \mathrm{~m}$. At each receiver antenna position, the received power was measured by Rohde\&Schwarz FSH6 100KHz-6GHz spectrum analyzer and FSH View. For $868 \mathrm{MHz}$, a $2 \mathrm{dBm}$ transmitted power level provided by the Programmable $1 \mathrm{GHz}$ synthesizer HM $8133-2$ has been used. However, for $2.5 \mathrm{GHz}$ and $5.8 \mathrm{GHz}$, the transmitter was connected to Rohde\&Schwarz $1 \mathrm{GHz}-40 \mathrm{GHz}$ signal generator providing $25 \mathrm{dBm}$ as transmitted power level so the RF communication can last for a longer distance. 
The next step was to lower the height of both transmitter and receiver simultaneously to $\mathrm{H}_{1}=0.2 \mathrm{~m}$ and apply the same procedure of the first step. After finishing with measurements, path loss was calculated using (1), and then (6) was used to estimate the break distance $d_{b}$ so the measured results can be compared with the proposed ones using $(2,3)$.

\subsection{RSSI Measurement Using Sensor Nodes Dedicated To Smart Agriculture}

In this experiment, we collected the Radio Signal Strength Indicator (RSSI) data in a short grass field to study how radio signal behaves in near ground conditions by using Libelium nodes working at $2.4 \mathrm{GHz} \mathrm{ZigBee}$ protocol. One node is intended for transmitting frames and the other one is for receiving and measuring the RSSI values. We changed as well the elevation of the nodes from the ground to observe the impact of the ground on the signal strength. Received signal strength indicator (RSSI) is a term used to describe a measurement of the power present in a received radio signal. RSSI is calculated at the radio chip on the base station and provides useful implication of wireless link quality [9]. To estimate the link quality between nodes in percentage from the RSSI values, formulas (7), (8) and (9) can be used.

$$
\begin{aligned}
& \text { If } \mathrm{RSSI} \geq-50 \mathrm{~dB} \text { then Quality }=100 \%, \\
& \text { Else If RSSI } \leq-100 \mathrm{~dB} \text { then Quality }=0 \% \text {, } \\
& \text { Else Quality }(\%) \approx 2 \times(\operatorname{RSSI}(\mathrm{dB})+100),
\end{aligned}
$$

At first, both nodes were fixed at a height where the elevation of the antenna is $\mathrm{H}_{2}=40 \mathrm{~cm}$ from the ground with $d_{1}=1 \mathrm{~m}$ as separation distance between them. The transmitter node was kept stationary and the receiver node was moved each time with $2 \mathrm{~m}$. RSSI was measured at each position of the receiver. After that, we putted the nodes just on the ground so the elevation of the antennas from the ground is $H_{1}=20 \mathrm{~cm}$, and repeated the same procedure as the first one.

\section{Results and Discussion}

\subsection{Indoor Experiment on Near Ground Path Loss in Near Field}

The measured data have been shown in Figure 1 and compared to the proposed model for the various frequency ranges.

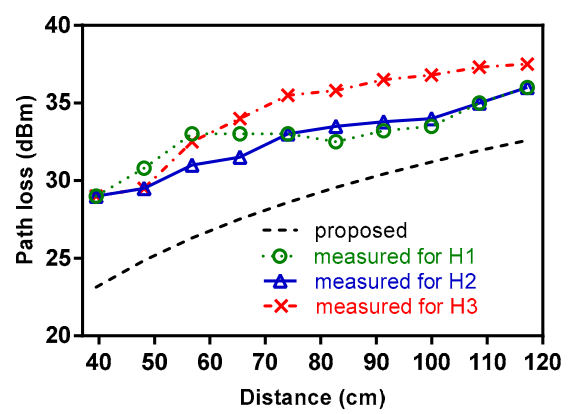

(a)

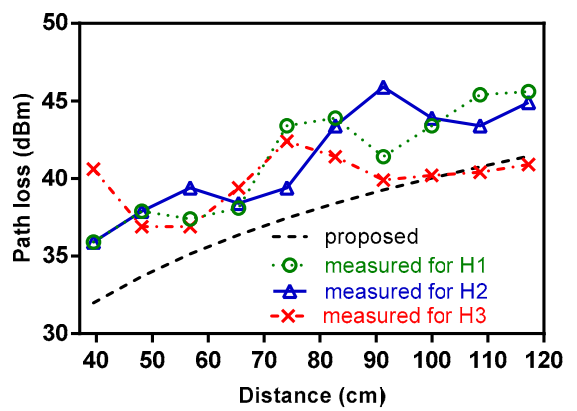

(b)

Figure 1. Cont. 


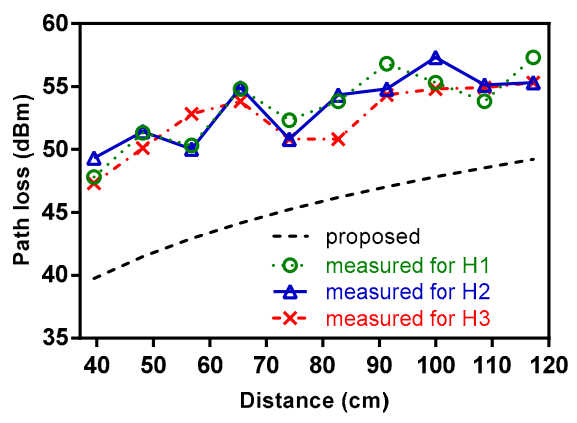

(c)

Figure 1. ComParison of the proposed model with path loss measurement results at frequency range (a) $\mathrm{F}_{1} ;$ (b) $\mathrm{F}_{2} ;$ (c) $\mathrm{F}_{3}$.

After Figure 1, we can see that the ground has no effect on the RF propagation in this case for all the three heights we tried. The losses exist in the path are almost equal even when the height of the transmitter and the receiver has been lowered. These results were expected because the maximum distance between the antennas is smaller than the calculated break distance, which is the minimum distance for the existence of near-ground path loss, for all the three frequency ranges using (6), so more than $60 \%$ of Fresnel zone is clear from obstructions. Just in Figure 1a, we can observe that the losses applied on the path are a little bigger for $\mathrm{H}_{3}$ because the break distance at that frequency in that height is close to the measured distance.

\subsection{Path Loss in Agriculture Fields Experiments}

The measured data in different experimented agriculture fields have been shown in Figure 2 and compared to the proposed model for the various frequency ranges.

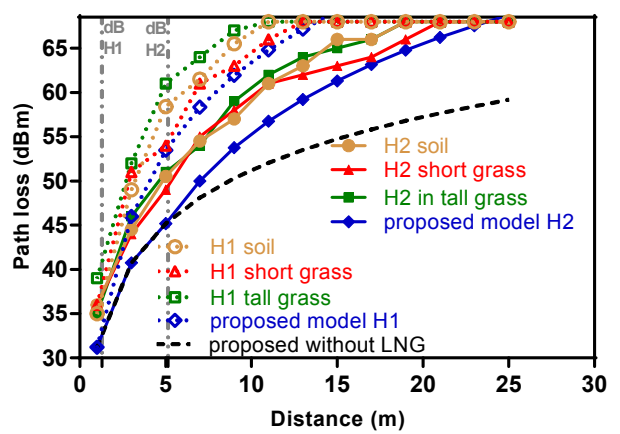

(a)

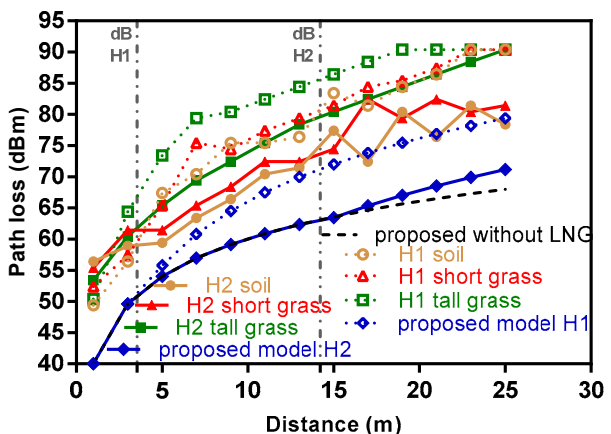

(b)

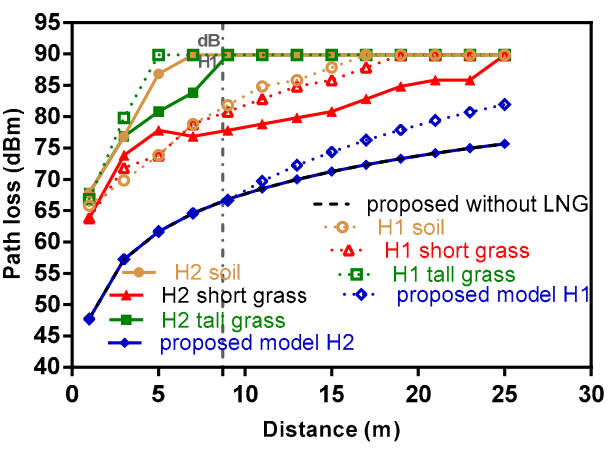

(c)

Figure 2. Path loss in agriculture fields at (a) $\mathrm{F}_{1}$; (b) $\mathrm{F}_{2}$; (c) $\mathrm{F}_{3}$. 
From Figure 2, it is evident that path loss values are increasing with decrease in antennas height. After analyzing these figures, we can observe that the transmitted signal has experienced the highest level of attenuation in tall grass field due to the length of the grass and the existence of more leafs that is obstructing the line of sight between the transmitter and the receiver, especially at $5.8 \mathrm{GHz}$, which showed a very poor performance not just in this field, but in all fields. As well, according to these figures, we can see that soil and short grass apply almost the same path loss on the transmitted signal in most cases. However, from Figure 2c, path loss caused by soil and short grass are similar at $\mathrm{H}_{1}$, but at the height of $\mathrm{H}_{2}$, the applied path loss by the soil on the transmitted signal are obviously higher than by short grass. Moreover, the communication between antennas in that case get lost at $7 \mathrm{~m}$ of distance between them, which is less comparing with when lowering the height of the antennas to $\mathrm{H}_{1}$. In all figures, we can see that the difference between the proposed and measured path loss is increasing with the increase of the frequency range, but also due due to ground irregularities and possible presence of scattering objects in the vicinity of the measurements.

Results have shown that $868 \mathrm{MHz}$ is typical for near ground nodes communications in agriculture fields, which is not a solution just for minimizing path losses, but also energy consumption, since the used transmitted power in case of $868 \mathrm{MHz}$ is $2 \mathrm{dBm}$ and $25 \mathrm{dBm}$ for $2.4 \mathrm{GHz}$ and $5.8 \mathrm{GHz}$.

\subsection{RSSI Measurement Using Sensor Nodes Dedicated to Smart Agriculture}

RSSI values were collected to observe the impact of the short grass field on WSN near ground nodes.

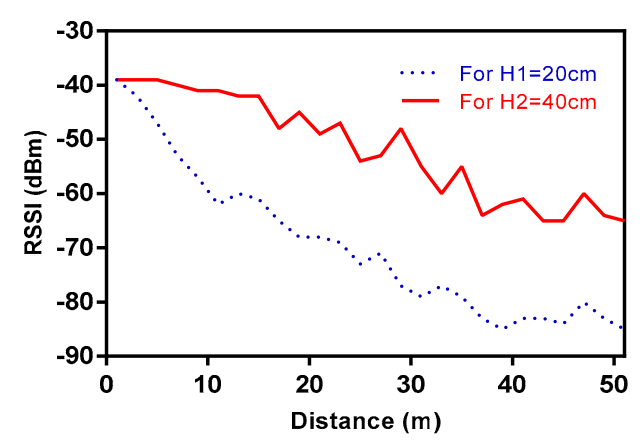

(a)

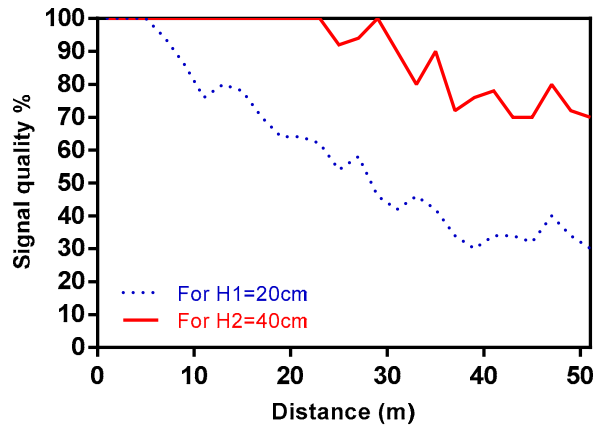

(b)

Figure 3. Difference at $\mathrm{H}_{1}$ and $\mathrm{H}_{2}$ between (a) RSSI values; (b) link quality.

From Figure 3a, it is clear that the height of the sensors from the ground can have a great effect on signal strength. It shows that the higher the position of the sensor from the ground, the stronger the signal strength which was expected through the experiments which have been done previously. Using (7), (8) and (9), we estimate the link quality between the waspmotes for both elevations from the RSSI measured values.

From Figure $3 b$ it is obvious that the link quality between the nodes is much better at $\mathrm{H}_{2}$. At this height, the signal quality remains $100 \%$ until $29 \mathrm{~m}$. However, at $\mathrm{H}_{1}$ the signal quality is decreasing from $5 \mathrm{~m}$. When the receiver node was placed $51 \mathrm{~m}$ away from the transmitter, we can say that the signal quality is perfect at this distance for $\mathrm{H}_{2}$ and not good for $\mathrm{H}_{1}$.

Acknowledgments: This research is supported by the Xunta de Galicia Predoctoral research grants 2017.

Conflicts of Interest: The authors declare no conflict of interest.

\section{References}

1. I.F. Akyildiz and Mehmet Can Vuran, Wireless Sensor Networks. Hoboken, NJ: Wiley, 2010. 
2. Chiara Buratti, Andrea Conti, Davide Dardari and Roberto Verdone, An overview on Wireless Sensor Networks Technology and evolution. Sensors 6869-6896, 2009.

3. D.J. Cook and S.K. Das, Smart Environments: Technology, Protocols, and Applications. Hoboken, NJ: Wiley, 2004.

4. J. Burrell, T. Brooke, and R. Beckwith. Vineyard computing: sensor networks in agricultural production. IEEE Pervasive Computing, 3(1):38-45, Jan-Mar 2004.

5. Kropff, M.; Wallinga, J.; Lotz, L. Modelling for Precision Weed Management. In Proceedings of Ciba Foundation Symposium 210-Precision Agriculture: Spatial and Temporal Variability of Environmental Quality, Chichester, UK, 27 September 2007; pp. 182-207.

6. Srinivasan, A. Handbook of Precision Agriculture: Principles and Applications; CRC: New York, NY, USA, 2006.

7. M I. Aslam and S.A. (Reza) Zekavat, New channel path loss model for near-ground antenna sensor networks. IET Wireless Sensor Systems, 2011.

8. Mauricio Rodriguez, Rodolfo Feick, Hector Carrasco, Reinaldo Valenzuela, Milan Derpich and Luciano Ahumada. Wireless Access Channels with Near-Ground Level Antennas. IEEE TRANSACTIONS ON WIRELESS COMMUNICATIONS, VOL. 11, NO. 6, JUNE 2012.

9. Lee, Ee Foong, Chen Wang and Li Xiao. A Study of Radio Signal Behaviors in Complex Environments.

(C2017 by the authors; licensee MDPI, Basel, Switzerland. This article is an open access article distributed under the terms and conditions of the Creative Commons Attribution (CC-BY) license (http://creativecommons.org/licenses/by/4.0/). 